\title{
SoSyM reflections of 2016: a journal status report
}

\author{
Geri Georg ${ }^{1}$ - Jeff Gray ${ }^{2}$ - Bernhard Rumpe ${ }^{3} \cdot$ Martin Schindler $^{3}$
}

Published online: 23 January 2017

(C) Springer-Verlag Berlin Heidelberg 2017

The past year has been an exciting time for SoSyM with the celebration of our 15th Anniversary, the addition of new Editors, and further collaboration with the MODELS conference (e.g., SoSyM awards at MODELS and the SoSyM Journal-first arrangement). The first issue of this new year's volume summarizes the status of SoSyM in terms of recent statistics and milestone events over the past year.
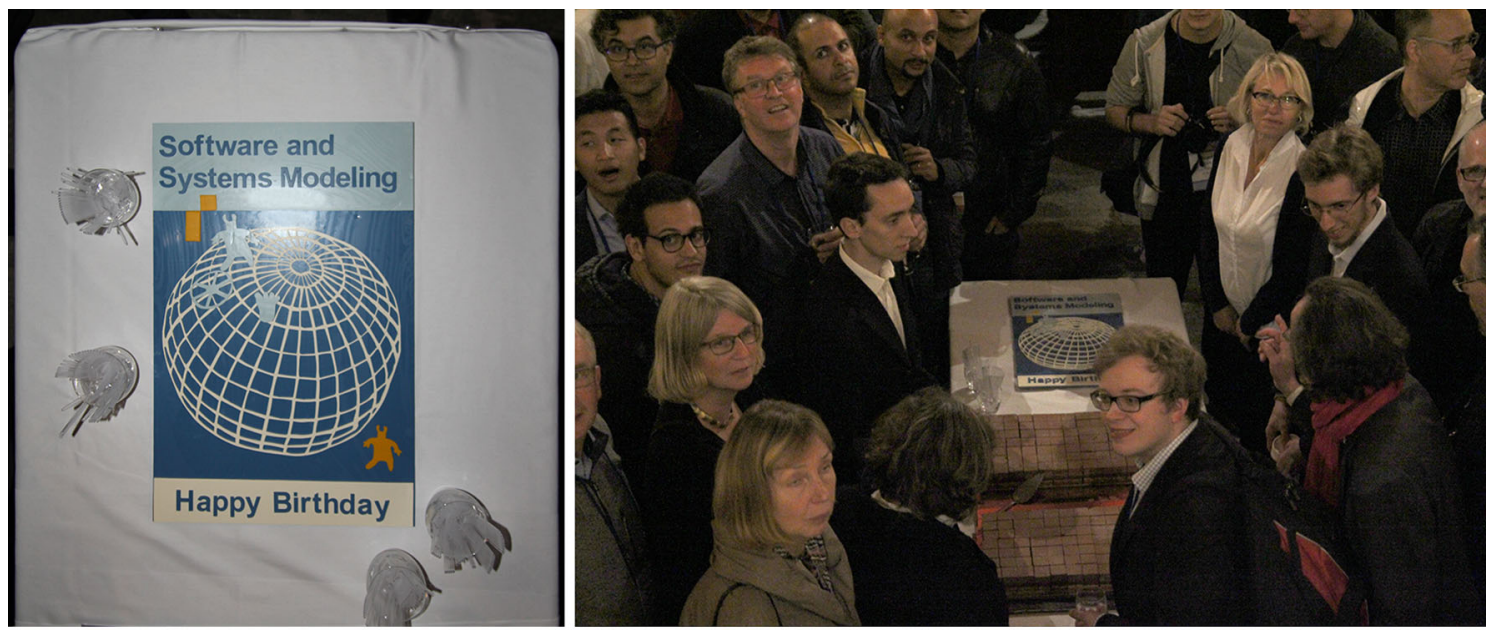

Photos of the 15th Anniversary Celebration at MODELS 2016

Martin Schindler

schindler@sosym.org

Geri Georg

georg@cs.colostate.edu

Jeff Gray

jeff.gray@ sosym.org

Bernhard Rumpe

bernhard.rumpe@sosym.org

Colorado State University, Fort Collins, CO, USA

2 University of Alabama, Tuscaloosa, AL, USA

3 RWTH Aachen University, Aachen, Germany

\section{6 summary statistics}

The submission and publication statistics for 2016 suggest an increased interest in SoSyM as a venue for authors in the modeling community.

Submission summary: There was a significant increase in the number of submissions in 2016 compared to 2015 . The 293 submissions in 2016 (compared to 198 in 2015) were made to the following categories: 190 Regular Papers, 35 Special Section Papers, 64 Theme Section Papers, and 4 Overview Papers. Corresponding to the increase in submissions, there was also an increase in the number of desk 
rejections, often due to a submission being out of scope, lacking depth, or missing an evaluation of the described work (suggestions for preparing a successful SoSyM submission are available in the Volume 15.4 Editorial, http:// dx.doi.org/10.1007/s10270-016-0558-5, archived at http:// www.sosym.org/editorials/). The acceptance rate for 2016 was $23.9 \%$ (21.8\% in 2015), with an average time to final decision at 127 days ( 145 days in 2015).

Impact and publication summary: The Impact Factor continued to fluctuate between consecutive years. The drop to 0.990 in the most recent calculation was down from 1.408 from the previous year, yet the 5-year Impact Factor of 1.497 suggests that SoSyM remains one of the top publication venues in Software Engineering, and the premier forum for the software and systems modeling community.

The number of paper downloads provided from the Springer SoSyM website represents the activity from the previous year (e.g., the 2016 year-end numbers are complete through 2015). The number of paper downloads reported for 2015 was slightly higher (42,346 downloads in 2015) than the previous report (41,357 downloads in 2014). Compared to 2010 (20,267 downloads) and 2011 (24,048 downloads), readership interest continues to improve.

Across the four issues of 2016, SoSyM published 53 total papers. The number of pages that was budgeted by Springer per issue in 2016 remained the same as in the previous year, at 294 pages. However, the overall number of pages published in 2016 was slightly higher than the budget. A summary of the publication statistics from 2016 and the past 15 years is provided in the following table.

\begin{tabular}{lll}
\hline & 2016 & $\begin{array}{l}\text { Since inception } \\
(2002-2015)\end{array}$ \\
\hline Regular Papers & 30 & 175 \\
Special Section Papers & 3 & 162 \\
Theme Section Papers & 11 & 87 \\
Editorials & 4 & 49 \\
Guest Editorials & 2 & 48 \\
Expert Voice Articles & 1 & 35 \\
Industry Voices Articles & 2 & 3 \\
Discussion/Overview Papers & 0 & 4 \\
Errata & 0 & 2 \\
TOTAL & 53 (1233 pages) & 565 (8836 pages) \\
\hline
\end{tabular}

\section{SoSyM's most influential paper awards}

The banquet dinner at MODELS 2016 in Saint Malo provided an opportunity to recognize the most influential SoSyM papers over the past 8 years. The selection was based on the ISI citation index among papers published in SoSyM from 2008-2015. More information about the awards can be found at: http://www.sosym.org/awards/.
The 8-year most influential regular paper award was given to the following authors:

- W. M. P. van der Aalst, Vladimir Rubin, H. M. W. Verbeek, Boudewijn F. van Dongen, Ekkart Kindler, and Christian W. Günther, "Process Mining: A Two-step Approach to Balance between Underfitting and Overfitting," In Journal on Software and Systems Modeling (SoSyM), 2010, vol. 9, no. 1, pp. 87-111. http://dx.doi. org/10.1007/s10270-008-0106-z

The 8-year most influential theme section paper award was given to the following authors:

- Kyriakos Anastasakis, Behzad Bordbar, Geri Georg, and Indrakshi Ray, "On Challenges of Model Transformation from UML to Alloy," In Journal on Software and Systems Modeling (SoSyM), 2010, vol. 9, no. 1, pp. 69-86. http:// dx.doi.org/10.1007/s10270-008-0110-3

\section{SoSyM's Journal-first papers presented at MODELS 2016}

For the second year, SoSyM is proud to collaborate with the MODELS conference organization in sponsoring the SoSyM Journal-first opportunity, which enables authors of recent SoSyM papers to present their work across the core conference sessions at MODELS. Through this collaboration, SoSyM authors have the opportunity to reach a broader audience to present their work, and the MODELS program is complemented by research talks that in their papers explore more depth and analytical and empirical evidence than a traditional conference submission can present. The MODELS 2016 program featured four articles published during the previous year (from July 2015 through June 2016). SoSyM looks forward to continuing this arrangement with MODELS 2017 organizers. The SoSyM Journal-first papers presented at MODELS 2016 were the following:

- Anna A. Kalenkova, Wil M. P. van der Aalst, Irina A. Lomazova, and Vladimir A. Rubin, "Process Mining using BPMN: Relating Event Logs and Process Models", In Journal on Software and Systems Modeling (SoSyM), Springer, 2015. http://dx.doi.org/10.1007/ s10270-015-0502-0

- Ana Ivanchikj, Cesare Pautasso, and Silvia Schreier, "Visual Modeling of RESTful Conversations with RESTalk", In Journal on Software and Systems Modeling (SoSyM), Springer, 2016. http://dx.doi.org/10.1007/ s10270-016-0532-2

- Vlad Acretoaie, Harald Störrle, and Daniel Strüber, "VMTL: A Language for End-user Model Transfor- 
mation", In Journal on Software and Systems Modeling (SoSyM), Springer, 2016. http://dx.doi.org/10.1007/ s10270-016-0546-9

- Katrina Falkner, Claudia Szabo, Vanea Chiprianov, Gavin Puddy, Marianne Rieckmann, Dan Fraser, and Cathlyn Aston, "Model-driven Performance Prediction of Systems of Systems", In Journal on Software and Systems Modeling (SoSyM), Springer, 2016. http://dx.doi.org/10. 1007/s10270-016-0547-8

Thanks to the authors for considering submitting their journal articles directly to SoSyM.

\section{Changes to the Editorial Board}

Several changes to the Editorial Board have occurred since the last issue. We thank Gerti Kappel and Jim Steel for their service to SoSyM as they retire as Editors. We also welcome five new Editors as we begin this new year and volume: Philippe Collet, Rob Pettit, Ina Schäfer, Andrzej Wasowski, and Manuel Wimmer. We appreciate their willingness to help in the SoSyM editorial responsibilities!

\section{Reviewers in 2016}

At the core of journal publishing is the deep effort provided by a community of reviewers. The quality of the literature available to the modeling community though SoSyM depends on the hard work of its reviewers. We appreciate the help from all of those who contributed a review in 2016! Below is a list of those who reviewed one or more papers for SoSyM in the last year. The complete list of reviewers can also be found on our website http://www.sosym.org/people/.

Silvia Abrahao, Rui Abreu, Rodina Ahmad, Mauricio Alferez, Shaukat Ali, Moussa Amrani, Daniel Amyot, Andreas Angerer, Anthony Anjorin, Stefan Appel, Ludovic Apvrille, Joanne Atlee, Marco Autili, Paris Avgeriou, Thomas Baar, Jean-Philippe Babau, Omar Badreddin, Mira Balaban, Muneera Bano, Ted Bapty, Olivier Barais, Jose Barateiro, Luciano Baresi, Jorge Barreiros, Chris Bates, Jürgen Becker, Steffen Becker, Kristian Beckers, Luca Berardinelli, Christian Berger, Richard Berntsson Svensson, Lorenzo Bettini, Robert Bill, Olivier Biot, Xavier Blanc, Arnaud Blouin, Karsten Boehm, Kirill Bogdanov, Andre Bondi, Etienne Borde, Frederic Boulanger, Fabrice Bouquet, Erwan Bousse, Premek Brada, Lionel Briand, Robert Brijder, David Broman, Manfred Broy, Achim Brucker, Achim Brucker, Jean-Michel Bruel, Hugo Bruneliere, Francois Bry, Peter Buchholz, Matthias Büchler, Didier Buchs, Barbora Buhnova, Tomas Bures, Erik Burger, Loli Burgueño, Fabien Campagne, Javier Luis Canovas Izquierdo, Anis Charfi,
François Charoy, De-Jiu Chen, Zheng Cheng, Antonio Cicchetti, Federico Ciccozzi, Robert Clarisó, Peter Clarke, Manuel Clavel, Sholom Cohen, Alan Colman, Isabelle Comyn-Wattiau, Kendra Cooper, Jonathan Corley, Vittorio Cortellessa, Stephen Cranefield, Ivica Crnkovic, Istvan Czibula, Andrea D'Ambrogio, Frederic Dadeau, Heinrich Daembkes, Zhen Ru Dai, Fabiano Dalpiaz, David Day, Guglielmo De Angelis, Juan de Lara, Erik de Vink, Julien DeAntoni, David Deharbe, Andreas Demuth, Birgit Demuth, Joerg Desel, Xavier Devroey, Stefano Di Alesio, Antinisca Di Marco, Elisabetta Di Nitto, Davide Di Ruscio, Jan Dietz, Juergen Dingel, Ivan Do Carmo Machado, Hubert Dubois, Mathias Ekstedt, Emelie Engström, Romina Eramo, Huseyin Ergin, Claudia Ermel, Juhan Ernits, Rik Eshuis, Huascar Espinoza, Ulrich Fahrenberg, Michalis Famelis, João Faria, Eduardo Fernandez, Eric Feron, Ulrich Frank, Jose Galindo, Ilias Gerostathopoulos, Aditya Ghose, Sudipto Ghosh, Holger Giese, Christoph Gladisch, Stefania Gnesi, Claude Godart, Martin Gogolla, Thomas Goldschmidt, Luís Gomes, Michael Gonzalez-Harbour, Javier González-Huerta, Carlos González, Steven Gordon, Jens Grabowski, Vincenzo Grassi, Jeff Gray, Marco Gribaudo, Emmanuel Grolleau, Alicia Grubb, Paul Grünbacher, John Grundy, Esther Guerra, Carlos Guerrero, Jens Gulden, Pragya Gupta, Jianda Han, Chihab Hanachi, Peter Harrison, Øystein Haugen, Regina Hebig, Reiko Heckel, Maritta Heisel, Constance Heitmeyer, Hadi Hemmati, Christopher Henard, Frank Hermann, Peter Herrmann, Mark Hills, Scott Hissam, Petr Hnetynka, Thai Son Hoang, Matthias Hoelzl, Berthold Hoffmann, Jennifer Horkoff, Akos Horvath, Pavel Hruby, Jerome Hugues, Benjamin Hummel, Mauro Iacono, Akram Idani, Alexei Iliasov, Ludovico Iovino, Muhammad Zohaib Iqbal, Luis Iribarne, Igor Ivkovic, Stefan Jablonski, Ethan Jackson, Paul Johannesson, Gert Johansson, Cliff Jones, Jan Juerjens, Ivan Jureta, Anna Kalenkova, Gregory Kapfhammer, Robert Karban, Holger Karl, Gabor Karsai, Christian Kästner, Timo Kehrer, Uzair Khan, Joerg Kienzle, Sybren Kinderen, Cornel Klein, Mathias Kleiner, Alexander Knapp, Christoph Knieke, Dimitrios Kolovos, Kostas Kontogiannis, Anna Magdalena Kosek, Xenofon Koutsoukos, Anne Koziolek, Anne Koziolek, Herb Krasner, Helmut Krcmar, John Krogstie, Thomas Kühne, Natalia Kushik, Juliana Küster Filipe Bowles, Marta Zofia Kwiatkowska, Marcel Kyas, Kevin Lano, Aron Laszka, Kung-Kiu Lau, Edward Lee, Tihamer Levendovszky, Hui Liu, Lin Liu, Malte Lochau, Delphine Longuet, Patricia López Martinez, Hong Lu, Jose Luis De La Vara, Mass Soldal Lund, Nuno Macedo, Ivano Malavolta, Frédéric Mallet, Shahar Maoz, Tiziana Margaria-Steffen, Leonardo Mariani, Miklos Maroti, Nicholas Matragkas, Tanja Mayerhofer, Nuria Medina-Medina, Julio Medina, Jan Mendling, Tom Mens, Mercedes Merayo, Jose Merseguer, Michael Michael, Zoltán Micskei, Gordana Milosavljevic, Mark Minas, Mehdi Mirakhorli, Naouel Moha, Pedro Molina, Sebastien Mosser, 
Jean-Marie Mottu, Laurent Mounier, Mohammad Mousavi, Ali Movaghar, Chokri Mraidha, Saad Mubeen, Gunter Mussbacher, John Mylopoulos, Clementine Nebut, $\mathrm{Cu}$ Nguyen, Phu Nguyen, Nan Niu, Manuel Núñez, Ileana Ober, Iliana Ober, Aida Omerovic, James Ortiz Vega, Frank Ortmeier, Sven Overhage, Richard Freeman Paige, Marc Pantel, Jose Parejo, Óscar Pastor López, Jan Peleska, Patrizio Pelliccione, Xin Peng, Marie-Agnes Peraldi-Frati, Antônio Pereira de Resende, Gilles Perrouin, Michaël Petit, Alexandre Petrenko, Dorina Petriu, Alfonso Pierantonio, Raquel Pillat, Geert Poels, Pascal Poizat, Andrea Polini, Hernán Ponce de León, Ansgar Radermacher, Eric Rapos, Andreas Rausch, Andreas Rausch, Henrique Rebelo, Atle Refsdal, Gil Regev, Gianna Reggio, Manfred Reichert, Hajo Reijers, Iris Reinhartz-Berger, Philipp Reinicke, Ralf Reussner, Andrew Reynolds, Leila Ribeiro, Stefani Rinderle-Ma, Jan Ringert, Charles Rivet, Harry Robinson, Genaína Rodrigues, David Ruiz, Ragnhild Runde, Mehrdad Saadatmand, Gunter Saake, Mehrdad Sabetzadeh, Shazia Sadiq, Rick Salay, Ashieh Salehi, Jesus Sánchez-Cuadrado, Pablo Sanchez, Chunyan Sang, Bernhard Schaetz, Ina Schieferdecker, Rainer Schmidt, Kurt Schneider, Pierre-Yves Schobbens, Andy Schürr, Martina Seidl, Lionel Seinturier, Bran Selic, Severine Sentilles, Bonita Sharif, Beijun Shen, Michael Shin, Samira Si-Said Cherfi, Joseph Sifakis, Jocelyn Simmonds, Marigianna Skouradaki, Oleg Sokolsky, Romina Spalazzese, Jonathan Sprinkle, Miroslav Staron, Friedrich Steimann, Matthew Stephan, Perdita Stevens, Serge Stinckwich, Ketil Stølen, Harald Störrle, Arnon Sturm, Yanchun Sun, Yu Sun, Gerson Sunye, Eugene Syriani, Janos Sztipanovits, Gabriele Taentzer, Tonatiuh Tapia-Flores, Jérémie Tatibouet, Stephan Thesing, Chouki Tibermacine, Matthias Tichy, Massimo Tisi, Andreas Tolk, Juha-Pekka Tolvanen, Louis-Marie Traonouez, Stavros Tripakis, Javier Troya, Dragos Truscan, T. H. Tse, Petr Tuma, Frédéric Tuong, Zoltán Ujhelyi, Mark Utting, Antonio Vallecillo, Wil van der Aalst, Frank van der Linden, Pieter Van Gorp, Dimitri Van Landuyt, Juan Manuel Vara, Tullio Vardanega, Gergely Varró, Yevgeniy Vorobeychik, Aneta Vulgarakis Feljan, Dennis Wagelaar,
Neil Walkinshaw, Shuai Wang, Andrzej Wasowski, Alan Wassyng, Barbara Weber, Joachim Wehler, Ran Wei, Hans Weigand, Tim Weilkiens, David Weiss, Alexander Wert, Matthias Wieland, Roel Wieringa, Erik Wilde, Manuel Wimmer, Michael Winikoff, Guido Wirtz, Marilyn Wolf, Burkhart Wolff, Katinka Wolter, Murray Woodside, Shin Yoo, Tingting Yu, Jelena Zdravkovic, Uwe Zdun, Gefei Zhang, Li Zhang, Wei Zhang, Yuting Zhao, Xiaoyun Zhu, Tewfik Ziadi, Alfred Zimmermann, Armin Zimmermann, and Steffen Zschaler.

\section{Content of this Issue}

In addition to four Regular Papers, this volume contains a Special Issue of the "11th International Conference on Software Engineering and Formal Methods" with Mario Bravetti, Robert M. Hierons, and Mercedes G. Merayo as guest editors. This volume also contains a Theme Issue on "Variability Modeling of Software-intensive Systems" with Andrzej Wasowski and Thorsten Weyer as guest editors.

The Regular Papers are:

- "Analyzing the Cognitive Effectiveness of the WebML Visual Notation" by Juan Manuel Vara, David Granada, Marco Brambilla, Veronica Bollati, and Esperanza Marcos.

- "Eugenia: Toward Disciplined and Automated Development of GMF-based Graphical Model Editors" by Dimitrios Kolovos, Antonio Garcia-Dominguez, Louis Rose, and Richard Paige.

- "Integrating Business Process Simulation and Information System Simulation for Performance Prediction" by Robert Heinrich, Philipp Merkle, Joerg Henss, and Barbara Paech.

- "An Empirical Approach toward the Resolution of Conflicts in Goal-oriented Models" by Jameleddine Hassine and Daniel Amyot. 